\title{
Exchange and the Coulomb blockade: Peak height statistics in quantum dots
}

\author{
Gonzalo Usaj and Harold U. Baranger \\ Department of Physics, Duke University, P.O.Box 90305, Durham North Carolina 27708-0305
}

(Dated: Received 29 Nov 2002)

\begin{abstract}
We study the effect of the exchange interaction on the Coulomb blockade peak height statistics in chaotic quantum dots. Because exchange reduces the level repulsion in the many body spectrum, it strongly affects the fluctuations of the peak conductance at finite temperature. We find that including exchange substantially improves the description of the experimental data. Moreover, it provides further evidence of the presence of high spin states $(S \geq 1)$ in such systems.

PACS numbers: 73.23.Hk, 73.40.Gk, 73.63.Kv
\end{abstract}

Transport properties of quantum dots (QDs) are strongly affected by interactions. A paradigmatic example is the Coulomb blockade (CB) of electron tunneling $\stackrel{1.2 .3}{\underline{*}}$ It occurs at low temperature when the thermal energy is smaller than the charging energy $\left(E_{C}\right)$ required to add an electron to the QD. The conductance is then blockaded, being restored only at specific values of the gate voltage $V_{g}$ of a nearby gate capacitively coupled to the QD. This leads to a series of sharp CB conductance peaks. The statistical properties of these peaks reflect the mesoscopic fluctuations of the (many-body) spectrum and wave-functions of the QD. In particular, they encode information about the non-trivial spin statistics that arises from the presence of exchange, the most important part of the residual interaction 3.4

Both the peak height distribution (PHD) and the peak spacing distribution have been studied in detail (see Ref. 2 for a review), though the latter has received most of the attention. This is mainly due to the fact that early measurements ${ }^{5.6}$ of the PHD were found to be in good agreement with the constant interaction (CI) model, 7.8 contrary to the case of the peak spacing. In this simple model of $\mathrm{CB}$, the $e-e$ interaction is assumed constant (given by $E_{C}$ ) and the fluctuations of the single particle properties described by random matrix theoryappropriate for chaotic (or diffusive) QDs.

A later experiment ${ }^{9}$ showed, however, significant deviations from the CI model. Namely, the peak height fluctuations were found to be smaller than expected in the entire experimental temperature range $\left(k_{\mathrm{B}} T=0.1-2 \Delta\right.$, with $\Delta$ the single particle mean level spacing). The authors attributed the discrepancy at high $T$ to the presence of dephasing but the origin of the low $T$ behavior was unclear-a recent calculation 10 showed that dephasing by itself cannot account for the high $T$ data either. It was then proposed 11 that the observed reduction of the fluctuations at low $T$ is related to the presence of spin-orbit coupling. Though the result is in good agreement with the low $T$ data, the assumed strength of the spin-orbit interaction requires some test-for instance, experimental data in Ref. 12 suggest that in small QDs (as used in Ref. 9) the effect of spin-orbit is rather weak.

So far, however, exchange- the main interaction effecthas not been considered. This contrasts with the case of the peak spacing distribution where it was shown to be crucial, as it leads to the appearance of non-trivial spin states in the QD $(S \geq 1)^{13.14 .15 .16}$ and enhances the effect of finite temperature $17, \overline{18}$
In this work we show that exchange also strongly affects the PHD. Furthermore, it accounts for most of the disagreement with the low $T$ data of Ref. 9, without including dephasing or spin-orbit coupling. Such agreement is yet another indication of the presence of high spin states in QDs.

A chaotic QD containing a large number of electrons can be described by the following Hamiltonian ${ }^{3.4 .15}$

$$
\hat{H}=\sum_{\alpha, \sigma} \varepsilon_{\alpha} \hat{n}_{\alpha, \sigma}+E_{C}(\hat{n}-\mathcal{N})^{2}-J_{S} \vec{S}^{2}
$$

where $\left\{\varepsilon_{\alpha}\right\}$, the single particle energies, are described by random matrix theory, $\mathcal{N}=C_{g} V_{g} / e$ describes the capacitive coupling to the control gate, $C_{g}$ is the dot-gate capacitance, $\vec{S}$ is the total spin operator, and $J_{S}$ is the exchange constant. The second and third terms in Eq. (1) describe the QD charging energy and the exchange interaction, respectively. We assume elastic transport and broken time-reversal symmetrytherefore we use the Gaussian Unitary Ensemble (GUE) unless otherwise stated.

In the regime $\Gamma \ll k_{\mathrm{B}} T, \Delta \ll E_{C}$, where $\Gamma$ is the total width of a level in the QD, the conductance near the $\mathrm{CB}$ peak corresponding to the $N-1 \rightarrow N$ transition is given by 18.19 .20 .21

$$
\begin{aligned}
G(x)= & \frac{e^{2}}{\hbar k_{\mathrm{B}} T} \sum_{i, j, \alpha, \sigma} \frac{\Gamma_{\alpha}^{L} \Gamma_{\alpha}^{R}}{\Gamma_{\alpha}^{L}+\Gamma_{\alpha}^{R}}\left|\left\langle\Psi_{j}^{N}\left|c_{\alpha, \sigma}^{\dagger}\right| \Psi_{i}^{N-1}\right\rangle\right|^{2} \\
& \times \frac{F_{\mathrm{eq}}(j) F_{\mathrm{eq}}(i)}{\left[\sqrt{F_{\mathrm{eq}}(j)}+\sqrt{F_{\mathrm{eq}}(i)}\right]^{2}} g_{j i}\left(x-x_{j i}^{*}\right)
\end{aligned}
$$

where $x=2 E_{C}\left[\mathcal{N}-\left(N-\frac{1}{2}\right)\right]$ and $x_{j i}^{*}$ is defined below. Here, (i) $\Gamma_{\alpha}^{L(R)}$ is the partial width of the single-particle level $\alpha$ due to tunneling to the left (right) lead, (ii) $\left\{\left|\Psi_{j}^{N}\right\rangle\right\}$ are the eigenstates of the QD with $N$ electrons, (iii) $F_{\text {eq }}(j)$ is the canonical probability that the eigenstate $j$ is occupied, and (iv)

$$
g_{j i}(x)=\frac{f\left(y_{j i}-x\right) f\left(y_{j i}+x\right)}{f\left(y_{j i}\right)^{2}}
$$

with $f(x)=\left(1+\exp \left[x / k_{\mathrm{B}} T\right]\right)^{-1}$ and $y_{j i}=$ $k_{\mathrm{B}} T \ln \left[F_{\mathrm{eq}}(j) / F_{\mathrm{eq}}(i)\right]$. Notice that $g_{j i}(x)$ reaches its maximum value at $x=0$. Let us denote by $\left\{E_{j}\right\}$ the eigenvalues of $\hat{H}$ without the charging energy term. Then, the contribution of the transition $i \rightarrow j$ to the conductance reaches 

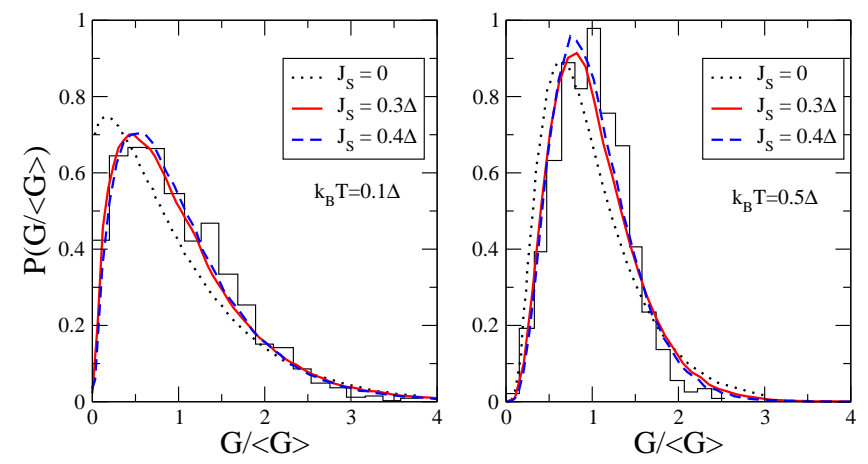

FIG. 1: Coulomb blockade peak height distribution at finite temperature. The theoretical distributions (lines) were obtained by numerically solving Eq. (2) for different values of the exchange constant $\left(J_{S}\right)$. The histograms correspond to the experimental data in Ref. 9. Notice that good agreement is obtained only after the addition of exchange.

its maximum when $x=x_{j i}^{*} \equiv E_{j}^{N}-E_{i}^{N-1}+y_{j i} / 2-E_{\mathrm{F}}$. Note the shift in the peak position due to finite temperature 17.18 .19 .22 .23 Since both $F_{\text {eq }}$ and the overlap $\left|\left\langle\Psi_{j}^{N}\left|c_{\alpha, \sigma}^{\dagger}\right| \Psi_{i}^{N-1}\right\rangle\right|^{2}$ depend on the spin of the two states involved in the transition, it is clear that this maximum is spin-dependent ${ }^{19,22,23,24}$

The eigenstates of the Hamiltonian (1) can be written as $\left|\Psi_{j}^{N}\right\rangle=\left|\left\{n_{\alpha}^{\prime}\right\}, S^{\prime}, S_{z}^{\prime}, k^{\prime}\right\rangle$, with $n_{\alpha}^{\prime}=n_{\alpha, \uparrow}^{\prime}+n_{\alpha, \downarrow}^{\prime}$ the occupation of the $\alpha$-th single particle state ${ }^{42}$ Since there is no energy dependence on $S_{z}^{\prime}$-Zeeman energy is neglected-the sum over $S_{z}, S_{z}^{\prime}$ and $\sigma$ in Eq. (2) can be easily carried out. One then gets $\frac{18.25}{2}$

$$
\sum_{\substack{S_{z}^{\prime}, k^{\prime} \\ S_{z}, k, \sigma}}\left|\left\langle\Psi_{S_{z}^{\prime}, k^{\prime}}^{N}\left|c_{\alpha, \sigma}^{\dagger}\right| \Psi_{S_{z}, k}^{N-1}\right\rangle\right|^{2}= \begin{cases}\left(2 S^{\prime}+1\right) N_{k} & \text { if } n_{\alpha}=0 \\ (2 S+1) N_{k^{\prime}} & \text { if } n_{\alpha}=1\end{cases}
$$

where $n_{\alpha}$ refers to the state with $N-1$ particles, $N_{k}\left(N_{k^{\prime}}\right)$ is the degeneracy associated with $k\left(k^{\prime}\right)^{43}$ and $\left|S^{\prime}-S\right|=\frac{1}{2}$.

In the special case of very low $T$, when only the ground state is relevant, we have $F_{\text {eq }}(j) \simeq 1 /\left(2 S^{\prime}+1\right), F_{\text {eq }}(i) \simeq$ $1 /(2 S+1)$, and $N_{k^{\prime}}=N_{k}=1$, so that the peak conductance is given by

$$
G_{\text {peak }}=\lambda_{S^{\prime}, S} \frac{2 e^{2}}{\hbar k_{\mathrm{B}} T} \frac{\Gamma_{\alpha}^{L} \Gamma_{\alpha}^{R}}{\Gamma_{\alpha}^{L}+\Gamma_{\alpha}^{R}}
$$

with

$$
\lambda_{S^{\prime}, S}=\frac{2\left(S^{\prime}+S\right)+3}{4\left(\sqrt{2 S^{\prime}+1}+\sqrt{2 S+1}\right)^{2}}
$$

where still $\left|S^{\prime}-S\right|=\frac{1}{2}$. Because the probability for the $S \rightarrow S^{\prime}$ transition depends on both the interaction strength $\left(J_{S}\right)$ and the statistics of the single particle spectrum, it turns out that the PHD depends, even at low $T$, on the fluctuations of both the wave-functions ( $\Gamma$ 's) and the many-body spectrum.

At finite temperature many transitions contribute to the conductance. We calculate the PHD by finding the maximum of

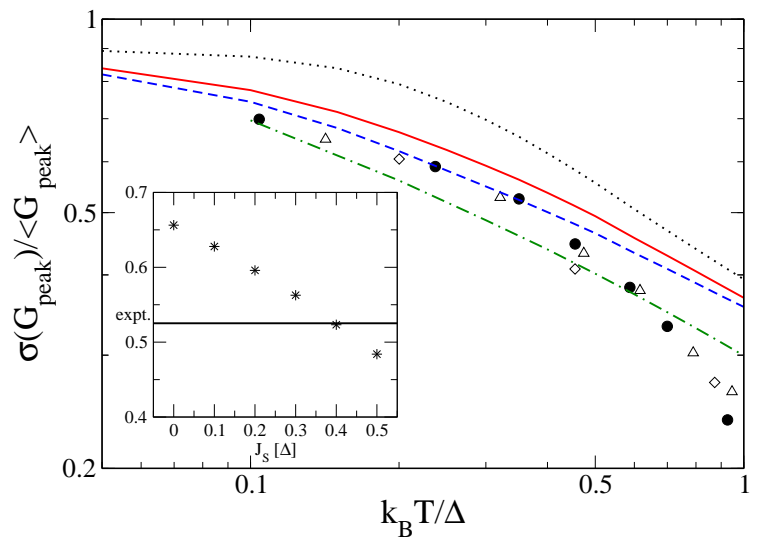

FIG. 2: Ratio of the root mean square to the mean of the conductance peak as a function of temperature. Different curves correspond to different values of exchange $J_{S}=0,0.3 \Delta$, and $0.4 \Delta$ (dotted, solid, and dashed lines, respectively). Symbols correspond to the data in Ref. 9. Notice the strong reduction of the fluctuation introduced by the exchange interaction at low temperature. The inset shows the decay of $\sigma\left(G_{\text {peak }}\right) /\left\langle G_{\text {peak }}\right\rangle$ as a function of $J_{S}$ at $k_{\mathrm{B}} T=0.35 \Delta$. At high $T$ the experimental data show a stronger suppression of the fluctuations than predicted in the strong inelastic regime for $J_{S}=$ $0.4 \Delta$ (dot-dashed line).

Eq. (2) numerically. According to random matrix theory, we describe the fluctuation of the widths $\Gamma_{\alpha}^{q}$ with $q=L, R$ by the Porter-Thomas distribution $P\left(\Gamma_{\alpha}^{q}\right) \stackrel{\alpha}{=} \bar{\Gamma}^{-1} \exp \left(-\Gamma_{\alpha}^{q} / \bar{\Gamma}\right)$, appropriate for the GUE. We keep states within an energy window of $\max \left(2 \Delta, 6 k_{\mathrm{B}} T\right)$ around the ground state.

Figure 1 shows the PHD for $k_{\mathrm{B}} T=0.1 \Delta, 0.5 \Delta$ and $J_{S}=0$, $0.3 \Delta, 0.4 \Delta$. The histograms correspond to the experimental data in Ref. 9. At low temperature the agreement is very good for the non-zero values of the exchange constant but clearly not for $J_{S}=0$ (CI model). This is indirect evidence for the presence of high spin states in QDs-we should point out though that spin-orbit leads to a similar effect if it is strong enough $\stackrel{11}{=}$ At higher temperature the presence of exchange improves the fit but not enough to fully account for the observed distribution. Nevertheless, it is clear that exchange substantially modifies the PHD and thus cannot be ignored.

Since an accurate measurement of the full PHD is quite demanding, a detailed comparison with theory is difficult. Instead, it is usually more convenient to look at the first few moments. In Ref. 9, the ratio of the root mean square and the mean value of the conductance peak height, $\sigma\left(G_{\text {peak }}\right) /\left\langle G_{\text {peak }}\right\rangle$, was measured as a function of temperature. It was found to be smaller than the value predicted by the CI model. Figure 2 compares the experimental data with our results for different values of $J_{S}$. The inset shows the $J_{S}$-dependence at a fixed temperature $\left(k_{\mathrm{B}} T=0.35 \Delta\right)$.

The improvement introduced by the exchange interaction at low temperature is evident. This can be easily understood as follows: because exchange reduces the level repulsion in the many body spectrum, the number of levels that contribute to the conductance at a given temperature increases and therefore the fluctuations are reduced-in fact, the im- 


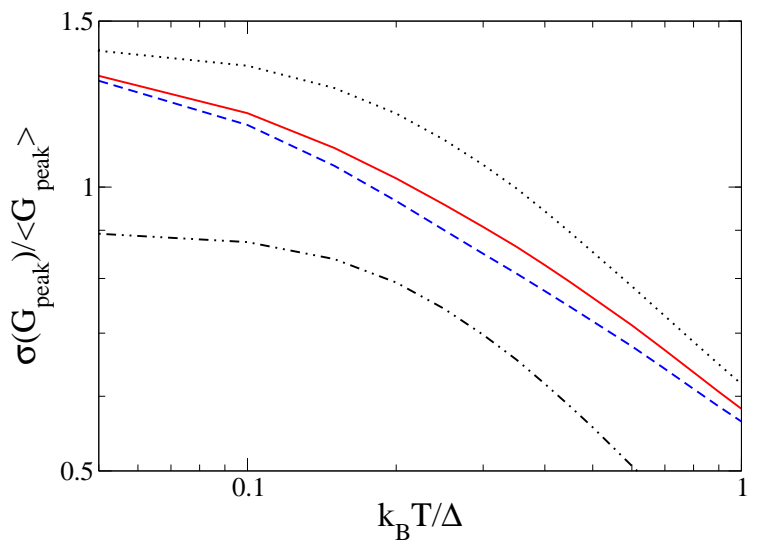

FIG. 3: Same as Figure 2 but for zero magnetic field (GOE). The GUE result for $J_{S}=0$ is included for comparison (dot-dot-dash line).

portance of the interplay between temperature and exchange was pointed out in Ref. 17. This is similar to the mechanism discussed in Ref. 11 where spin-orbit coupling reduces the repulsion in the single particle spectrum. At very low temperature, Eq. (5) predicts an enhancement of the fluctuations compared to the $\mathrm{CI}$ model result, $\sigma\left(G_{\text {peak }}\right) /\left\langle G_{\text {peak }}\right\rangle=$ $\left[\left\langle\lambda_{S^{\prime}, S}^{2}\right\rangle /\left\langle\lambda_{S^{\prime}, S}\right\rangle^{2} \times 9 / 5-1\right]^{1 / 2} \geq 2 / \sqrt{5}$. However, this enhancement is negligible since $\left\langle\lambda_{S^{\prime}, S}^{2}\right\rangle /\left\langle\lambda_{S^{\prime}, S}\right\rangle^{2} \simeq 1$ for typical values of the interaction. We also checked that the effect on Fig. 2] of non-universal corrections ${ }^{3}$ to the Hamiltonian (1) is very small, consistent with the results for the peak spacing distribution where they mainly affect the shape of the distribution. $17.18,26$

Our model shows a significant deviation from the experimental data for $k_{\mathrm{B}} T \geq 0.5 \Delta$. It is tempting to attribute this to the presence of inelastic processes, which we have not taken into account so far. Notice that after exchange is included, dephasing only needs to account for a (small) fraction of the reduction of the fluctuations. Accounting for an arbitrary inelastic rate requires solving a master equation for the transition probabilities, which is not a simple task ${ }^{19.27}$ Instead, we calculate the peak conductance in the strong inelastic regime, ${ }^{28}$ where electrons inside the QD are assumed to be in thermal equilibrium. In practice, this means that instead of calculating the thermal average of $\Gamma_{\alpha}^{L} \Gamma_{\alpha}^{R} /\left(\Gamma_{\alpha}^{L}+\Gamma_{\alpha}^{R}\right)$-see Eq. (2) - , one first calculates the thermal average of the couplings $\left\{\Gamma_{\alpha}^{q}\right\}$ and then the ratio, $\left\langle\Gamma^{L}\right\rangle\left\langle\Gamma^{R}\right\rangle /\left\langle\Gamma^{L}+\Gamma^{R}\right\rangle$. This is expected to be a lower bound for the experimental data. Figure 2 shows that this is not the case. The reason for this is unclear.

A direct comparison between our results and the experimental data seems to suggest that $J_{S} \simeq 0.4 \Delta$ (see inset in Figure 2). This value of the interaction is bigger than what one would estimate from an RPA calculation of the screened potential for the experimental density $\left(J_{S} \simeq 0.3 \Delta\right){ }^{3.18} \mathrm{At}$ present it is not clear to us whether corrections beyond RPA are required, ${ }^{29.30}$ or if other effects such as spin-orbit ${ }^{11}$ need to be included.

It is interesting, then, to consider ways to distinguish between the exchange and spin-orbit scenarios. In the latter

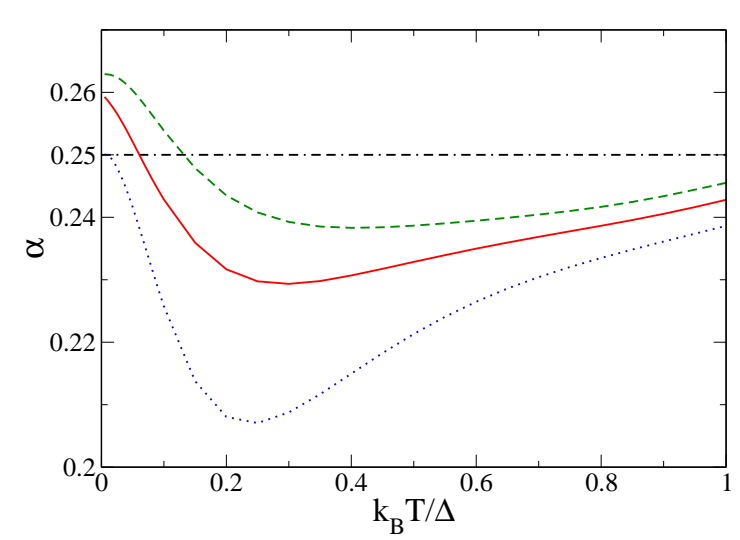

FIG. 4: Relative change in the mean conductance upon breaking time-reversal symmetry [ $\alpha$, see Eq. (4)] as a function of temperature. Different curves correspond to different values of the exchange constant: 0 (dotted), $0.3 \Delta$ (solid), and $0.4 \Delta$ (dashed). Notice that $\alpha$ is increased by exchange and, in particular, that it is bigger than 0.25 for $T \rightarrow 0$ when $J_{S}>0$.

case, it was predicted ${ }^{11}$ that an in-plane magnetic field will restore the level repulsion and increase the fluctuations. The behavior is the opposite in the absence of a perpendicular magnetic field, where the system will evolve (as the in-plane field is increased) from the orthogonal to the unitary ensemble (see Figure 3) ${ }^{11}$ The same general trend is also valid in the exchange-dominated scenario because a large enough parallel field will always drive the system towards the strong spin-orbit regime. ${ }^{31}$ Therefore, the only difference between the two scenarios would be the specific dependence of $\sigma\left(G_{\text {peak }}\right) /\left\langle G_{\text {peak }}\right\rangle$ on the magnitude of the parallel field.

A sharper distinction between the two scenarios could be made by changing the electron density $n_{e}$ in the QD. On the one hand, an increase of $n_{e}$ will slightly decrease $J_{S}$ and therefore enhance the fluctuations (see Figures 2 and 3 . On the other hand, increasing $n_{e}$ increases the magnitude of the spin-orbit coupling ${ }^{12,32}$ and so decreases the conductance fluctuations-according to Ref. 12 , we assume that the initial value of the spin-orbit coupling is smaller than the one required to complete the crossover described in Ref. 11. Therefore, the sign of $\partial\left[\sigma\left(G_{\text {peak }}\right) /\left\langle G_{\text {peak }}\right\rangle\right] / \partial n_{e}$ at low $T$ is different for each scenario - a sharp distinction.

It is worth pointing out that an independent measurement of $J_{S}$-obtained, for instance, by measuring the spin distribution ${ }^{33.34 .35 .36}$ — would be a direct way to rule out or quantify the effect of spin-orbit coupling and exchange.

Finally, we study the effect of exchange on the relative change of the mean value of the conductance upon breaking time-reversal symmetry,

$$
\alpha=1-\frac{\left\langle G_{\text {peak }}\right\rangle_{\mathrm{GOE}}}{\left\langle G_{\text {peak }}\right\rangle_{\mathrm{GUE}}} .
$$

Very recently, the temperature dependence of this quantity was measured by Folk et al. $\frac{37}{3}$ wo used it to estimate the dephasing time $\left(\tau_{\phi}\right)$ in weakly coupled QDs. ${ }^{38}$ This estimate is based on the deviation of the experimental value of $\alpha$ from 
the one expected within the CI model in the elastic regime, namely, $\alpha \simeq 0.25$ for both $k_{\mathrm{B}} T \ll \Delta$ and $k_{\mathrm{B}} T \gg \Delta .39$ For intermediate temperatures $\alpha$ is slightly smaller than 0.25 because of the difference in the spectral fluctuations of the two ensembles ${ }^{10.40}$

Figure 4 illustrates the temperature dependence of $\alpha$ for three different values of $J_{S}$. Notice that $\alpha$ increases monotonically with $J_{S}$ in the full temperature range. That is, exchange enhances $\alpha$. In particular, the bound $\alpha \leq 0.25$ is no longer valid at low temperature. ${ }^{18}$ This can be verified using Eq. (5),

$$
\alpha \simeq 1-\frac{3}{4} \frac{\left\langle\lambda_{S^{\prime}, S}\right\rangle_{\mathrm{GOE}}}{\left\langle\lambda_{S^{\prime}, S}\right\rangle_{\mathrm{GUE}}}>0.25
$$

This enhancement originates in the difference between the spin distributions in the two ensembles-high spin states are more likely to occur in the GOE. Also note that the effect of the spectral fluctuations is substantially reduced. As a result, $\alpha$ remains closer to 0.25 than in the CI model. Unfortunately, because of large statistical errors in the experimental data, ${ }^{37}$ this difference could not be discerned.

Since inelastic processes reduce $\alpha, 27,28$ the bigger the difference between the elastic result and the measured value of $\alpha$ the shorter $\tau_{\phi}$. Then, it is clear that neglecting exchange overestimates $\tau_{\phi}$. Though we do not expect the difference to be large, it could be relevant when deciding whether or not there is a saturation of $\tau_{\phi}$.

In summary, we have studied the effect of the exchange interaction on the peak height distribution. We found that it strongly affects the distribution at finite temperature. Our results indicate that the experimental data present signatures of high spin states and suggest that $J_{S} \gtrsim 0.3 \Delta$ for the dots of Ref. 9 .

We appreciate helpful discussions with K. Held, E. Eisenberg, P. Brouwer, and B. L. Altshuler. GU acknowledges partial support from CONICET (Argentina). This work was supported in part by the NSF (DMR-0103003). After submission of this paper, we learned of Ref. 41 which contains some overlapping material.
${ }^{1}$ L. P. Kouwenhoven, C. M. Marcus, P. L. McEuen, S. Tarucha, R. M. Westervelt, and N. S. Wingreen, in Mesoscopic Electron Transport, edited by L. L. Sohn, L. P. Kouwenhoven, and G. Schön (Kluwer, New York, 1997), pp. 105-214.

2 Y. Alhassid, Rev. Mod. Phys. 72, 895 (2000).

3 I. L. Aleiner, P. W. Brouwer, and L. I. Glazman, Phys. Rep. 358, 309 (2002).

${ }^{4}$ I. L. Aleiner and L. I. Glazman, Phys. Rev. B 57, 9608 (1998).

5 A. M. Chang, H. U. Baranger, L. N. Pfeiffer, K. W. West, and T. Y. Chang, Phys. Rev. Lett. 76, 1695 (1996).

6 J. A. Folk, S. R. Patel, S. F. Godijn, A. G. Huibers, S. M. Cronenwett, C. M. Marcus, K. Campman, and A. C. Gossard, Phys. Rev. Lett. 76, 1699 (1996).

7 R. A. Jalabert, A. D. Stone, and Y. Alhassid, Phys. Rev. Lett. 68, 3468 (1992).

8 Y. Alhassid and A. Wobst, Phys. Rev. B 65, 041304 (2002), also found good agreement using a model of spinless interacting particles.

${ }^{9}$ S. R. Patel, D. R. Stewart, C. M. Marcus, M. Gökçedağ, Y. Alhassid, A. D. Stone, C. I. Duruöz, and J. S. Harris, Phys. Rev. Lett. 81, 5900 (1998).

10 T. Rupp, Y. Alhassid, and S. Malhotra, Phys. Rev. B 65, 193304 (2002).

${ }^{11}$ K. Held, E. Eisenberg, and B. L. Altshuler (2002), condmat/0208177.

12 D. M. Zümbuhl, J. B. Miller, C. M. Marcus, K. Campman, and A. C. Gossard, Phys. Rev. Lett. 89, 276803 (2002).

13 P. W. Brouwer, Y. Oreg, and B. I. Halperin, Phys. Rev. B 60, R13977 (1999).

${ }^{14}$ H. U. Baranger, D. Ullmo, and L. I. Glazman, Phys. Rev. B 61, R2425 (2000).

15 I. L. Kurland, I. L. Aleiner, and B. L. Altshuler, Phys. Rev. B 62, 14886 (2000).

16 D. Ullmo and H. U. Baranger, Phys. Rev. B 64, 245324 (2001).

17 G. Usaj and H. U. Baranger, Phys. Rev. B 64, 201319(R) (2001).

18 G. Usaj and H. U. Baranger, Phys. Rev. B 66, 155333 (2002).

${ }^{19}$ C. W. J. Beenakker, Phys. Rev. B 44, 1646 (1991).
${ }^{20}$ Y. Meir and N. S. Wingreen, Phys. Rev. Lett. 68, 2512 (1992).

${ }^{21}$ H. M. Pastawski, Phys. Rev. B 46, 4053 (1992).

${ }^{22}$ L. I. Glazman and K. A. Matveev, Pis'ma Zh. Éksp. Teor. Fiz. 48, 403 (1988), [JETP Lett. 48, 445 (1988)].

${ }^{23}$ H. Akera, Phys. Rev. B 59, 9802 (1999).

${ }^{24}$ D. Weinmann, W. Häusler, and B. Kramer, Phys. Rev. Lett. 74, 984 (1995).

25 R. Pauncz, Spin Eigenfunctions: Construction and Use (Plenum Press, New York, 1979).

26 Y. Alhassid and S. Malhotra, Phys. Rev. B 66, 245313 (2002).

${ }^{27}$ E. Eisenberg, K. Held, and B. L. Altshuler, Phys. Rev. Lett. 88, 136801 (2002).

${ }^{28}$ C. W. J. Beenakker, H. Schomerus, and P. G. Silvestrov, Phys. Rev. B 64, 033307 (2001).

${ }^{29}$ H. Jiang, H. U. Baranger, and W. Yang, Phys. Rev. Lett. 90, 026806 (2003).

30 Y. Oreg, P. W. Brouwer, X. Waintal, and B. I. Halperin (2001), and references therein, cond-mat/0109541.

31 J. A. Folk, S. R. Patel, K. M. Birnbaum, C. M. Marcus, C. I. Duruöz, and J. S. Harris, Phys. Rev. Lett. 86, 2102 (2001).

32 J. B. Miller, D. M. Zümbuhl, C. M. Marcus, Y. B. Lyanda-Geller, D. Goldhaber-Gordon, K. Campman, and A. C. Gossard (2002), cond-mat/0206375.

33 P. Jacquod and A. D. Stone, Phys. Rev. Lett. 84, 3938 (2000).

${ }^{34}$ I. L. Kurland, R. Berkovits, and B. L. Altshuler, Phys. Rev. Lett. 86, 3380 (2001).

35 J. A. Folk, C. M. Marcus, R. Berkovits, I. L.Kurland, I. L.Aleiner, and B. L. Altshuler, Physica Scripta T90, 26 (2001).

${ }^{36}$ S. Lindemann, T. Ihn, T. Heintzel, W. Zwerger, K. Ensslin, K. Maranowski, and A. C. Gossard, Phys. Rev. B 66, 195314 (2002).

37 J. A. Folk, C. M. Marcus, and J. S. Harris, Phys. Rev. Lett. 87, 206802 (2001)

38 B. L. Altshuler, Y. Gefen, A. Kamenev, and L. S. Levitov, Phys. Rev. Lett. 78, 2803 (1997).

39 Y. Alhassid, Phys. Rev. B 58, 13383 (1998).

${ }^{40}$ K. Held, E. Eisenberg, and B. L. Altshuler, Phys. Rev. B 66, 
033308 (2002).

41 Y. Alhassid and T. Rupp (2002), cond-mat/0212126.

42 An extra quantum number $k^{\prime}$ is required when the number of singly occupied levels is bigger than 2 (see Ref. 25).
${ }^{43}$ If $N_{S}$ is the number of singly occupied levels, then $N_{k}=$ $\left(\begin{array}{c}N_{S} \\ \frac{1}{2} N_{S}-S\end{array}\right)-\left(\begin{array}{c}N_{S} \\ \frac{1}{2} N_{S}-S-1\end{array}\right)$. 